\title{
El uso de las tecnologías de la información y la comunicación (TIC) en los centros de salud:
}

\author{
la visión de los profesionales en Cataluña, España
}

Martí Oliver-Mora(a)

Lupicinio Iñiguez-Rueda(b)

Oliver-Mora M Iñiguez-Rueda L. The use of information and communication technologies (ICTS) in health centers: the practititoners' point of view in Catalonia, Spain. Interface (Botucatu). 2017; 21(63):945-55.

The aim of this research is to identify experiences using information and communication technologies (ICTs) that are able to improve the public management of health centers in Catalonia (Spain), as well as being generated bottom-up by primary health care practitioners. We have compiled these experiences through eleven semi-structured interviews with primary care physicians and nurses. The result is a set of experiences that can improve public management in health centers through a) greater interaction between patients and primary health care providers; $b$ ) more patient-centred medical care; and c) greater participation and involvement of patients in their own health situation.

Key words: Public health. Health centers. Communications media. Diffusion of innovation. Qualitative research.
Esta investigación tiene por objetivo la identificación de experiencias en el uso de las tecnologías de la información y la comunicación (TIC) que sean capaces de mejorar la gestión publica de los centros de salud en Cataluña (España), y que hayan sido impulsadas "desde abajo hacia arriba" por parte de los profesionales de la atención primaria. Hemos recopilado estas experiencias mediante la realización de once entrevistas semi-estructuradas a profesionales médicos/as y enfermeros/ as de la atención primaria. El resultado constituye un conjunto de experiencias que pueden mejorar la gestión pública de los centros de salud a través de a) una mayor interacción entre pacientes y profesionales de la atención primaria; b) una medicina más centrada en el paciente; y c) una mayor participación e implicación de los pacientes en su estado de salud.

Palabras clave: Salud pública. Centros de salud. Medios de comunicación. Difusión de innovaciones. Investigación cualitativa.

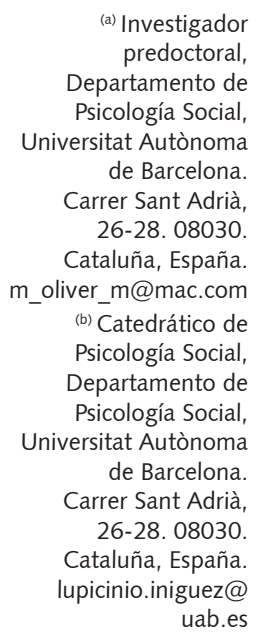

(a) Investigador predoctoral, Departamento de Psicología Social, Universitat Autònoma de Barcelona. Carrer Sant Adrià, 26-28. 08030. Cataluña, España. m_oliver_m@mac.com (b) Catedrático de Psicología Social, Departamento de Psicología Social Universitat Autònoma de Barcelona. Carrer Sant Adrià, 26-28. 08030 Cataluña, España. lupicinio.iniguez@ uab.es 


\section{Introducción}

Identificar experiencias capaces de mejorar la gestión de las instituciones públicas ha sido tradicionalmente -y sigue siendo a día de hoy- uno de los principales objetivos de políticos, gestores, y de todos aquellos profesionales interesados en mejorar la prestación de servicios de este tipo de instituciones. Sin embargo, la situación de recortes presupuestarios a la que están sometidas actualmente gran parte de las instituciones públicas en Cataluña -así como en el conjunto de España-, está provocando que "mejorar la gestión de las instituciones públicas" se entienda simplemente como una forma de reducir sus costes y aumentar su productividad.

Una de las instituciones públicas donde se están aplicando ampliamente los recortes presupuestarios en Cataluña son los centros de salud, de los que nos ocuparemos en este artículo. Estos recortes, extendidos al conjunto del sistema público de salud, están suponiendo el cierre constante de ambulatorios, así como la retirada de numerosas ambulancias y camas en los hospitales. Al mismo tiempo, las movilizaciones de los profesionales del ámbito de la salud ponen de manifiesto las condiciones laborales cada vez más precarias a las que se les está sometiendo, y es por este motivo que resulta necesario problematizar hasta qué punto las políticas ejecutadas hasta el día de hoy son las únicas medidas posibles.

Por el contrario, a partir de la consecución del objetivo planteado en la presente investigación, éste es, identificar experiencias en el uso de las tecnologías de la información y la comunicación (TIC) que sean capaces de mejorar la gestión pública de los centros de salud en Cataluña, y que hayan sido impulsadas "desde abajo hacia arriba" por parte de los profesionales de la atención primaria, argumentaremos que mejorar la gestión de los centros de salud no pasa por recortar sus presupuestos ni las condiciones laborales de sus trabajadores, sino por potenciar aquellas experiencias que contribuyan a una mejora de sus dinámicas, tanto sociales como organizativas o técnicas.

De forma paralela, la progresiva introducción de las TIC en nuestro día a día está poniendo en evidencia las potencialidades de que disponen este tipo de herramientas para facilitar, entre otras cosas, nuevas formas de comunicación e interacción social ${ }^{1-3}$. En el ámbito concreto de la salud, la introducción de las TIC ha dado lugar a diferentes plataformas de interacción social -ya sea entre pacientes, entre profesionales de la salud, o entre pacientes y profesionales de la salud--, que están flexibilizando la concepción tradicional de la asistencia sanitaria. Así, el uso de las TIC en los centros de salud está haciendo que algunos de los problemas de las consultas presenciales, como los escasos minutos de que disponen los pacientes para interactuar con los profesionales médicos y de enfermería, se vean parcialmente solventados con las posibilidades de que disponen las TIC para ampliar estos canales de comunicación.

\section{Método}

Hemos identificado las experiencias en el uso de las TIC en los centros de salud de Cataluña mediante la realización de once entrevistas semi-estructuradas de una duración de entre 45 y 90 minutos a informantes clave y a impulsores de experiencias con potencial de innovación seleccionados a través de la aplicación de la técnica de bola de nieve. Así, en primer lugar hemos entrevistado a profesionales expertos en atención primaria. Estas primeras entrevistas nos han proporcionado el contacto de profesionales de la salud que están impulsando experiencias, mayoritariamente relacionadas con la aplicación de las tecnologías Web 2.0 en el ámbito de la salud. En un segundo lugar, hemos entrevistado a estos profesionales, con un guion de entrevista focalizado en los objetivos y las características de cada una de las experiencias en cuestión. El Cuadro 1 resume los apartados que han estructurado estas entrevistas y los objetivos que han perseguido. 
Cuadro 1. Apartados y objetivos entrevistas a impulsores de experiencias

\begin{tabular}{|l|l|}
\hline \multicolumn{1}{|c|}{ Apartado } & \multicolumn{1}{c|}{ Objetivo } \\
\hline 1. Definición y objetivo & Definir la experiencia según las palabras de sus impulsores y los objetivos perseguidos. \\
\hline 2. El proceso de creación & $\begin{array}{l}\text { Identificar cómo surgió inicialmente la idea y los actores implicados, sus motivaciones } \\
\text { personales y profesionales, las fuentes de inspiración, las principales dificultades en su } \\
\text { desarrollo, y cómo ha modificado su día a día como profesionales de la salud. }\end{array}$ \\
\hline $\begin{array}{l}\text { 3. Funcionamiento y } \\
\text { respuesta }\end{array}$ & $\begin{array}{l}\text { Caracterizar las TIC utilizadas en el desarrollo de la experiencia, el personal encargado de su } \\
\text { ejecución, las necesidades a las que intenta dar respuesta, y perfil del paciente destinatario. }\end{array}$ \\
\hline 4. Resultados obtenidos & $\begin{array}{l}\text { Dar cuenta de los cambios que ha propiciado en el día a día de los pacientes, el grado de } \\
\text { aceptación o rechazo por parte de otros profesionales sanitarios, sugerencias de mejora, y el } \\
\text { grado de cumplimiento de los objetivos establecidos. }\end{array}$ \\
\hline 5. De cara al futuro & \begin{tabular}{l} 
Identificar nuevos retos de cara al futuro. \\
\hline 6. Otras experiencias
\end{tabular} \\
$\begin{array}{l}\text { Identificar las principales áreas de innovación en atención primaria, así como otras experiencias } \\
\text { de Salud 2.0 relevantes. }\end{array}$ \\
\hline
\end{tabular}

Asimismo, y siguiendo a Barney Glaser y Anselm Strauss ${ }^{4}$, hemos analizado la información recopilada en las entrevistas mediante el método de las comparaciones constantes. Este método consiste en un proceso continuo de comparación entre las diferentes categorías que van emergiendo inductivamente a partir del análisis del material obtenido en el trabajo de campo, según la importancia que les atribuyan sus protagonistas.

Hemos dividido este proceso de análisis en cuatro fases. En primer lugar, la fase de codificación de apertura ha consistido en establecer un primer grupo de códigos iniciales en los cuales hemos plasmado aquellos aspectos que, dada una primera impresión, nos han parecido más relevantes para las personas entrevistadas. Así, gran parte de los códigos de apertura han consistido en una clasificación general de las experiencias identificadas, por ejemplo, entre aquellas experiencias impulsadas por parte de los profesionales de la atención primaria y que hacen uso de las TIC para relacionarse con los pacientes, y aquellas que las utilizan para relacionarse con los profesionales de la atención secundaria.

En segundo lugar, en la fase de segmentación, hemos utilizado la codificación de apertura previamente definida, para dividir el texto general en partes, las cuales han formado unidades de significado y unidades de trabajo. El objetivo ha sido constituir unidades segmentadas que den cuenta por ellas solas de los significados contextuales, y que a la vez puedan ser tratadas de forma intensiva.

En tercer lugar, la fase de codificación focalizada ha consistido en crear subcódigos dentro de los códigos de apertura que sean capaces de dotarlos de una coherencia interna. Las reflexiones realizadas a lo largo del trabajo de campo, anotadas esquemáticamente en una libreta, nos han servido para realizar esta codificación focalizada. Así, por ejemplo, una clasificación realizada por uno de los profesionales entrevistados consistente en dividir las experiencias en gestión de los centros de salud según su carácter informativo o terapéutico, nos ayudó posteriormente en la aplicación de códigos focalizados, enfatizando en el carácter informativo o terapéutico de las demás experiencias.

Paralelamente, tanto los códigos de apertura como los subcódigos de uno y otro segmento han sido comparados según todas las combinaciones posibles, estableciendo así las diferencias o similitudes existentes entre códigos y subcódigos de un y otro segmento. Finalmente, hemos clasificado los diferentes segmentos que contienen unos mismos temas, creando así lo que se ha llamado códigos fundamentados, es decir, códigos con una alta coherencia interna y con mucha diferencia respecto a los otros códigos fundamentados.

En cuarto y último lugar, la fase de (re)contextualización ha implicado trazar el camino que ha seguido cada segmento y cada subcódigo hasta formar los códigos fundamentados. Esta cuarta fase es la que explica por qué los diferentes códigos fundamentados han terminado constituyéndose de una determinada manera, a la vez que permite entrever más claramente en qué difieren de los demás. 


\section{Resultados y discusión}

La introducción de las TIC en el sistema sanitario ha dado lugar a una batería de nuevos conceptos que han abierto el campo de la reflexión acerca de las potencialidades y limitaciones de la aplicabilidad de estas tecnologías en el día a día de los profesionales de la salud y de los pacientes. Mientras que de un modo más genérico, el término "eSalud" hace referencia al uso de Internet en el ámbito de la salud, con el más restrictivo "Salud 2.0" se da un paso más allá al introducir las nuevas oportunidades que ofrece la Web 2.05. El término "Web 2.0", atribuido generalmente a Tim O'Reilly ${ }^{6}$, hace referencia a aquellas aplicaciones web wikis, blogs, tagging, etc.- que posibilitan un uso más interactivo, participativo y personalizado por parte de los usuarios.

En este sentido, también en Cataluña se han introducido recientemente herramientas propias tanto de lo que hemos definido como eSalud, como de Salud 2.0. Es así que retomando nuestro objetivo de investigación, en las páginas que siguen vamos a dar cuenta de las experiencias en el uso de las TIC que sean capaces de mejorar la gestión pública de los centros de salud, y que hayan sido impulsadas 'desde abajo hacia arriba' por parte de los profesionales de la atención primaria, que hemos podido identificar en Cataluña. Concretamente, nos centraremos en cómo la introducción de las TIC puede mejorar la interacción entre pacientes y profesionales de la atención primaria, fomentar una medicina más centrada en el paciente, y potenciar la toma de decisiones por parte de los pacientes en relación a su estado de salud.

\section{Carácter sociópeto}

Tal vez resulte una obviedad decir que las TIC tienen un carácter sociópeto, es decir, que fomentan la comunicación y la interacción social. Es más, puede que fuese la función original por la cual nacieron. Lo que resulta más difícil es entender por qué en un contexto como el actual en el que las TIC inundan nuestra cotidianidad, nos encontramos tan alejados de ellas cuando se trata de relacionarnos con el sistema sanitario. No sólo entre los pacientes y el sistema sanitario, también existe una escasa comunicación, por ejemplo, entre los profesionales de la atención primaria y los profesionales de la atención secundaria.

En este sentido, y a pesar del impacto que ha supuesto el uso del correo electrónico en nuestro día a día, no existía hasta hace muy pocos años un proyecto específicamente destinado a potenciar el uso generalizado de esta herramienta como una vía de interacción entre los pacientes y los profesionales de la atención primaria. De todas formas, esta ausencia no impidió que muchos profesionales optaran por potenciar este medio de comunicación por su cuenta:

[...] consultas electrónicas, yo las utilizo, pero lo utilizo a la chita callando y desde hace muchos años, porque en teoría no se pueden utilizar las redes porque es secreto, no son seguras, no están codificadas, no está encriptado y todo esto, pero yo hace muchos años que las estoy recogiendo. Las administrativas del centro lo hacen, hace mucho tiempo que están recogiendo los correos electrónicos de los pacientes, y si el paciente autoriza [...] yo lo hago por correo electrónico, obviamente pidiendo antes permiso al paciente, le digo: "mira, qué te parece si envío un e-mail al [nombre del doctor] y le consulto tu electro", entonces el paciente lo autoriza, yo lo pongo en el historial clínico, y digo: "acuerdo con el paciente enviar por correo electrónico al [nombre del doctor] para consultar". (Margot)(c)

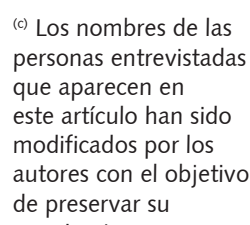


Sin embargo, y como apunta el extracto anterior, el hecho de que los correos electrónicos no estén encriptados, ha limitado la generalización de esta herramienta como una vía de comunicación segura. Fue precisamente como respuesta a la inseguridad que supone la comunicación a través de correo electrónico sin encriptación, que la gerencia del ámbito de atención primaria de Barcelona Ciudad, puso en marcha, entre los años 2009 y 2011, una prueba piloto para implementar un sistema de comunicación mediante correo electrónico llamado eConsulta. Este proyecto consistió en poner en contacto los pacientes y los profesionales de la atención primaria a través de una plataforma virtual. Esta plataforma permitía que los pacientes realizaran preguntas y trámites de diferente índole a través del correo electrónico, evitando así la realización de consultas presenciales que perfectamente se podían solucionar telemáticamente. En su gran mayoría, se trata de funciones informativas como puede ser el hecho de haber olvidado algunos datos referentes a la prescripción de medicamentos, informar de los resultados favorables de una analítica, tramitar una baja médica, o enviar una determinada documentación.

Sin embargo, un salto cualitativo en la comunicación entre los pacientes y los profesionales de la atención primaria viene dado por la conducción, por parte de estos últimos, de blogs destinados a los pacientes. En lo referente al carácter sociópeto, y en contraposición a otras herramientas como el correo electrónico o el teléfono, los blogs ponen a disposición de los pacientes informaciones complementarias como el calendario y el horario de consulta del médico, o la opción de pedir hora de consulta directamente a través del blog. Además, el mismo blog puede ser un canal para poner en manos de los pacientes diferentes informaciones de carácter general. Este es el caso, por ejemplo, de uno de los médicos entrevistados, que a través del blog informaba a sus pacientes, entre otras cosas, de los cambios en los horarios de apertura de su centro de salud, del cambio de ubicación que sufrió recientemente, de cómo los recortes podían afectar la prestación de determinados servicios, o incluso de si iría o no a trabajar durante una jornada de huelga general, con su respectiva justificación. Sin embargo, las mayores potencialidades de que disponen los blogs para pacientes no versan tanto sobre su carácter estrictamente sociópeto, como sobre su función de fomentar una actitud más crítica en el paciente en relación a su estado de salud, como abordaremos en el próximo apartado.

Una de las herramienta de comunicación que está creciendo con mayor rapidez en el sistema sanitario catalán es ECOPIH (Herramienta de Comunicación entre Primaria y Hospitalaria, en catalán), una comunidad virtual que pone en contacto los especialistas en atención primaria con los especialistas en atención secundaria. Sin embargo, su principal virtud no reside en el simple hecho de poner en contacto los profesionales del centro de salud con los del hospital, puesto que dicha comunicación hace tiempo que se viene realizando a través de otros medios. El valor añadido de ECOPIH reside en que todos los médicos inscritos en la plataforma, sea cual sea su especialidad, pueden acceder a los comentarios realizados por parte de sus compañeros y participar en la discusión. Así pues, y aunque la idea es que en un primer momento el profesional de atención primaria sea respondido por parte de aquel profesional de la atención secundaria al que habría derivado el paciente, esta dinámica de pregunta-respuesta puede ser fácilmente complementada por los comentarios de otros profesionales, potenciando así el debate y la participación. Más aún, las dudas planteadas pueden ir acompañadas tanto de imágenes, como de documentos (artículos, protocolos, etc.), que pueden ayudar a comprender el caso y/o a difundir recursos de interés general.

Tal y como apuntan Kathryn Horner, Ed Wagner et al. ${ }^{7}$, la ausencia de comunicación entre los profesionales de la atención primaria y los de la atención secundaria comporta un conjunto de errores en las derivaciones que normalmente se traducen en un aumento tanto del malestar de los pacientes, como de la ineficacia del sistema sanitario. Entre estos errores se encuentran básicamente las derivaciones innecesarias, las derivaciones erróneas, es decir, dirigidas al especialista que no corresponde con las necesidades del paciente, y la ausencia de la información requerida por parte del profesional hospitalario para llevar a cabo el tratamiento. Sin embargo, para estos autores las comunidades de consulta virtual entre los profesionales de la atención primaria y los de la atención secundaria - como es el caso de ECOPIH -, se presentan como una óptima solución para mejorar la calidad de las derivaciones. 


\section{Carácter participativo}

Fomentar la participación de los pacientes en su estado de salud hace referencia a romper con la idea según la cual un paciente es alguien que, ante todo, tiene paciencia. A nivel mediático, casos como el de Dave de Bronkart, un paciente diagnosticado de un tipo de cáncer con un promedio de supervivencia de seis meses, y que desde entonces se ha convertido en un militante a favor de un mayor empoderamiento por parte de los pacientes, han hecho emerger la figura del e-Paciente ${ }^{8}$. Siguiendo a Kersin Adahl ${ }^{9}$, podemos entender por e-Paciente aquel paciente que usa Internet para mantenerse informado y para aprender sobre aquellos aspectos relacionados con la salud que le afectan directamente. En esta misma línea, el e-Paciente es alguien que busca comunicarse, ya sea con otros pacientes, ya sea con los profesionales médicos y de enfermería, a fin de disponer de un mayor conocimiento sobre su estado de salud. Es de este modo que la figura del e-Paciente se mueve en una doble dirección: por una parte, busca potenciar la capacidad y el empoderamiento de los pacientes para tomar decisiones médicas en su día a día, y por otra parte, promueve una medicina más participativa y centrada en el paciente.

El primero de estos dos aspectos ha sido desarrollado ampliamente por Don Nutbeam ${ }^{10,11}$, quien lo ha dado a conocer con el nombre de Health literacy. Este autor distingue tres niveles de Health literacy. En primer lugar, la functional literacy constituye la capacidad básica de saber leer y escribir con la suficiente fluidez para poder actuar eficazmente en situaciones de poca complejidad. En segundo lugar, la interactive literacy da un paso más allá al implicar la capacidad de los pacientes de participar activamente en el sistema sanitario, extraer información de interés en relación a sus inquietudes, o comunicarse con médicos y pacientes para intercambiar opiniones. Finalmente, la critical literacy supone disponer de los conocimientos suficientes para poder discernir entre la información disponible, analizarla críticamente y tener la capacidad de aplicarla en el día a día para ejercer un mayor control sobre el propio estado de salud. Sin embargo, este autor reconoce que la fuerza de la health literacy no reside tanto en la capacidad del paciente de procesar la información sanitaria, como de desarrollar una mayor implicación en su estado de salud.

De todos modos, y aunque necesario, no es suficiente que el paciente se sensibilice y apueste por una actitud más crítica en relación a su estado de salud, sino que al mismo tiempo es importante que el sistema público de salud acompañe estos cambios. Es precisamente a este rol de apertura del sistema sanitario hacia una mayor implicación de los pacientes a lo que hace referencia el segundo de los aspectos mencionados anteriormente. Para Kersin Adahl ${ }^{9}$, una medicina centrada en el paciente implica pasar de un paradigma en que el paciente es visto como un objeto al que aplicar un tratamiento, a verlo como un sujeto, en el sentido de que sus experiencias subjetivas deben igualmente tomarse en consideración a la hora de aplicar las decisiones e intervenciones médicas. Para que esta medicina centrada en el paciente sea efectiva, resulta obvia la necesidad de potenciar una relación más estrecha entre pacientes y profesionales de la atención primaria. Es en este sentido que las TIC pueden contribuir a esta labor potenciando una mayor participación de los pacientes en el sistema sanitario.

Una de las experiencias capaces de mejorar el grado de participación de los pacientes en su estado de salud consiste en la conducción de blogs para pacientes por parte de los profesionales de la atención primaria. Como hemos avanzado anteriormente, la conducción de estos blogs da lugar a una relación con los pacientes que de otro modo sería inimaginable. Más allá del carácter estrictamente sociópeto, que como apuntamos en el apartado anterior, pueden cumplir estas herramientas, los blogs de médicos ponen a disposición de toda la comunidad virtual una gran cantidad de recursos para la formación permanente, contribuyendo así a mejorar la health literacy de los pacientes. De esta manera, las entradas realizadas por parte de los médicos en sus blogs, sirven a menudo para que sus propios pacientes obtengan una mayor información sobre su estado de salud, o sobre los tratamientos a los que van a someterse:

[...] una entrada que realicé [en el blog] sobre el uso indebido que hacen de la toxina botulínica, que es esta, la del famoso Bótox, pues ahora tengo a una paciente que le envían a que la pruebe, y le pude decir: "mira, aquí está escrito", así que yo supongo que sí, que poco a poco, 
el hecho de que vayas dando informaciones también para un sector de la población cada vez más elevado, pues que puede ser útil. (Antonio)

De todas formas, no es esta condición de intermediarios de la información lo que mejor define la actitud de muchos de estos médicos. Ante el aumento del fenómeno que algunos profesionales de la atención primaria llaman "Dr. Google", caracterizado por el hecho de que el paciente busca información en Internet sobre una determinada enfermedad sin ningún tipo de intermediario -más allá del criterio que establece el servidor Google para dar respuesta a la búsqueda en cuestión-, podría parecer que los profesionales de la atención primaria deben cumplir con una función de intermediarios para que el acceso a dicha información por parte de los pacientes esté mejor dirigida.

Sin embargo, la función de estos blogs para pacientes está más cercana a lo que Gunthen Eysenbach ${ }^{12}$ ha llamado apomediación (apomediation), a medio camino entre la intermediación (intermediation) y la ausencia de intermediación (disintermediation). Para este autor, la apomediación es propia de las posibilidades que ofrece la Web 2.0, y consiste en una estrategia de búsqueda de información en la que el paciente confía menos en la voz de los expertos, los tradicionales intermediarios de la información, para pasar a tomar en mayor consideración la voz de los legos, por ejemplo, mediante los comentarios de otros pacientes acerca de determinadas informaciones. Es así que la transmisión unidireccional de información profesional médico-paciente, es substituida por una trasmisión mediada por otros legos que se sitúan entre el trasmisor original de la información y el receptor final. En suma, podemos decir que la apomediación puede contribuir a substituir el paternalismo que caracteriza gran parte de las relaciones entre los profesionales de la atención primaria y los pacientes, por una actitud de mayor empoderamiento por parte de estos últimos:

[...] el objetivo es que el paciente tenga información, pero no la que yo le puedo proporcionar, que él piense [...] sobre todo esto, pasar de una información direccional del médico de "usted tiene que hacerse estas pruebas, usted tal, tal", a un modelo de compartir, que es lo que se llama Web 2.0. Pues compartir información y que el paciente tenga herramientas para responsabilizarse. Si el paciente acepta, entonces ningún problema, pero que él sepa. (Joaquín)

De esta forma, y como se apunta en el extracto precedente, el carácter participativo de los blogs viene dado, especialmente, por su componente de Web 2.0 que permite al paciente interactuar tanto con el conductor del blog, como con otros pacientes, contribuyendo así a romper con su estatus de agente pasivo.

Finalmente, nos gustaría mencionar el caso de las comunidades virtuales de pacientes, otra herramienta que directa o indirectamente fomenta la participación y la apomediación en la transmisión de información médica para pacientes. En Cataluña, debe destacarse la experiencia de Forumclínic, una comunidad virtual coordinada por profesionales de centros hospitalarios y de atención primaria cuyo objetivo consiste en aumentar la autonomía de los pacientes en relación a su salud aprovechando las oportunidades que ofrecen para ello las nuevas TIC.

La plataforma está organizada a partir de las diez comunidades, pertenecientes cada una de ellas a una enfermedad crónica diferente. Como en las otras experiencias de este tipo, la opción más interesante de Forumclínic consiste en el foro social existente para cada enfermedad, moderado en este caso por un equipo de profesionales sanitarios de la misma Corporación Sanitaria Clínic. Es precisamente a través de este foro social que Forumclínic adquiere un carácter de apomediación, dando la posibilidad a los pacientes de convertirse en agentes activos en interacción tanto con los profesionales médicos como con otros pacientes. En cada una de las comunidades, el usuario puede acceder, asimismo, a información específica sobre su enfermedad de interés, a noticias de actualidad sobre la misma, y a una sección de vídeos relacionados.

En suma, las comunidades virtuales de pacientes pueden verse como una herramienta tanto para el fomento de la health literacy, potenciando la implicación y la sensibilización del paciente en relación a su estado de salud, como para el desarrollo de una medicina más centrada en el paciente. Así, se trata de un contexto donde los pacientes pueden expresar libremente sus malestares, a la vez que dialogar acerca de ello con otros pacientes y profesionales sanitarios. 


\section{Experiencias desde las trincheras}

Resulta llamativo que algunas de las experiencias abordadas en los apartados anteriores -como ECOPIH o la conducción de blogs para pacientes-, no cuentan con un apoyo institucional en términos de presupuesto o de tiempo. Por el contrario, se trata de experiencias que sobreviven gracias a la voluntad de sus impulsores, o como resaltan algunos de ellos, 'por amor al arte'. Así, estas experiencias emergen y se desarrollan gracias al valor que les otorgan sus impulsores; un valor muy ligado tanto al hecho de haber surgido desde abajo, como al hecho de haber nacido como respuesta a una necesidad detectada en el día a día por parte de los profesionales de la atención primaria.

Este es el caso, por ejemplo, de ECOPIH, una experiencia surgida de la voluntad de dos profesionales de la atención primaria que coordinan, moderan y administran esta plataforma virtual de comunicación entre la atención primaria y la secundaria:

[ECOPIH] es una cosa que ha surgido desde abajo, es decir, que ha salido "desde las trincheras", que se dice; se ha detectado, y nosotros mismos hemos puesto una solución que pensamos que es la más adecuada a lo que nosotros necesitamos, y ya está. ¿Esto qué quiere decir? Que donde lo presentamos, todo el mundo cree que irá muy bien, todo el mundo, obviamente siempre hay gente que no, pero en general la respuesta siempre es buenísima, y la gente confía que esto irá bien, y también desde la organización, pero digamos, la organización te apoya y nos ayuda a que esto vaya adelante, pero no ha hecho una apuesta decidida de decir: "vayamos a saco con esto, confiemos en este proyecto, y démosles todas estas herramientas" (Sergio).

Del mismo modo, fueron los propios profesionales los que detectaron la necesidad de mejorar la comunicación entre la atención primaria y la secundaria, y los que decidieron movilizar los recursos que tenían a su alcance para dar una respuesta a esta deficiencia que limitaba su día a día como profesionales de la salud:

Nosotros en primaria siempre tenemos el problema de que quieres consultar una cosa con el especialista, y este tema no está muy bien resuelto. Existen consultas presenciales de ver al especialista cada mes, dos veces al mes o cada semana, depende; hay un servicio que tiene correo electrónico, pero esto ya es una forma un poco más informal. Las consultas presenciales, claro, tienen limitaciones: tienes que estar en un momento concreto, depende de que el especialista no se haya tomado un día libre, si tú tienes una duda tal vez tienes que esperarte hasta el mes que viene porque vino ayer, después si lo quieres llamar tienes que llamar al hospital, encontrarlo a veces es complicado, y esto [ECOPIH] te permite tener esta comunicación fácil (Sergio).

Asimismo, los blogs para pacientes son el resultado de la voluntad de abrir nuevas vías de comunicación entre los profesionales de la atención primaria y los pacientes. Sin embargo, no existen abundantes propuestas por parte de los equipos de atención primaria de crear una plataforma unitaria para suplir estas necesidades. Contrariamente, debemos entender su emergencia como el fruto de la emprendeduría individual de algunos de estos profesionales:

[...] una de las motivaciones íntimas es que en el equipo como tal, no había una gran proyección exterior y veías que como equipo, si tenía que esperar que se moviera todo, no lo lograríamos. Ahora, el director me dice: "a ver cuándo hacemos un blog para el equipo", y yo le digo: "ningún problema, cuando quieras". (Antonio)

Este es el caso, igualmente, de otro de los médicos que lidera un blogs para pacientes. Sin embargo, en esta ocasión, la decisión de poner en marcha un blog para pacientes no puede entenderse sin su experiencia previa en la dinamización de un blog para profesionales de la atención primaria:

[...] ahora hace tres años que empecé con el tema. Los médicos tenemos mucha información y pienso: "¿dónde guardo todos estos documentos que nos llegan?", que es un problema que tenemos en el día a día. Entonces creé, en primer lugar, el que sería un blog profesional de interrelación entre médicos. [...] fue hace un año que dije: “ahora ya tengo la información 
interprofesional, y también quiero que el paciente tenga información útil, ¿cómo lo hago?" En un blog, porque es una herramienta gratuita y fácil (Joaquín).

Finalmente, la experiencia de eConsulta constituye un caso paradigmático de experiencia surgida desde abajo y que ha terminado por institucionalizarse. Aún tratándose de una experiencia impulsada por parte de la gerencia del área de Barcelona Ciudad del Instituto Catalán de la Salud, el hecho de que su gerente fuese un médico de la atención primaria, buen conocedor de la realidad de los centros de salud, hace que podamos hablar de eConsulta como de una experiencia surgida para dar respuesta a las necesidades detectadas por parte de los profesionales de la atención primaria. En palabras de una de sus impulsoras:

[eConsulta] surgió mientras yo estaba en la gerencia de atención primaria de Barcelona, y era porque había mucha gente que utilizaba el correo electrónico para comunicarse con sus pacientes, pero no creían que fuera la forma más segura, ni estaban cómodos dando su correo a los pacientes, entonces empezamos a preparar el proyecto [...]. (Eva)

El éxito de eConsulta como una experiencia de interacción mediante correo electrónico entre pacientes y profesionales de la atención primaria en el área de Barcelona Ciudad, ha facilitado la implantación por parte del Instituto Catalán de la Salud de una nueva plataforma virtual con unas características muy similares para el conjunto de las áreas sanitarias de Cataluña, representando de esta forma la generalización de su uso.

\section{Consideraciones finales}

En 1992, Ezekiel y Linda Emanue ${ }^{13}$ publicaron un influyente artículo en que describían cuatro modelos de relación médico-paciente. Estos cuatro tipo de relaciones iban del modelo paternalista al modelo deliberativo, pasando por el modelo informativo y el interpretativo. A partir de estos cuatro tipos ideales, los autores marcaban un recorrido, el hilo conductor del cual era la medida en que se fomentaba la implicación del paciente en el control de su estado de salud, siendo el modelo paternalista el más cerrado a este tipo de implicación, y el modelo deliberativo el más abierto. Finalmente, abogaban por el modelo deliberativo, argumentando que la autonomía, la posibilidad de discutir con el médico, la persuasión frente a la imposición, y la medicina como un todo frente a la medicina de la abusiva fragmentación de los cuerpos, debían ser valores al alza en el sistema sanitario.

Sin embargo, en estos más de veinte años las posibilidades de que disponen los pacientes para interactuar y participar en el sistema sanitario han cambiado enormemente. Así, y desde entonces, han ido surgiendo varios conceptos como "e-Paciente", "Health literacy", "apomediación", entre otros, que expresan las posibilidades que ofrecen las TIC para implicar en mayor medida los pacientes en su estado de salud.

Concretamente, la introducción de las TIC en el sistema sanitario puede contribuir a mejorar diferentes aspectos, entre los cuales nos gustaría resaltar: a) la relación de confianza entre los pacientes y los profesionales de la atención primaria; b) la formación de los pacientes entorno a su estado de salud; c) la constitución de redes de apoyo entre pacientes; d) la autonomía y la capacidad decisoria de los pacientes; e) una atención sanitaria más personalizada; f) la colaboración entre los profesionales sanitarios; y g) la calidad de las derivaciones de la atención primaria a la atención secundaria.

De todas formas, y en el contexto actual de recortes presupuestarios realizados en Cataluña, las administraciones públicas no se están mostrando muy interesadas en potenciar este tipo de iniciativas. Es por este motivo que las experiencias identificadas, e impulsadas 'desde abajo hacia arriba' por parte de los profesionales de la atención primaria, obtienen mayor relevancia. Su implantación da cuenta de la voluntad de muchos de los profesionales de la atención primaria de continuar innovando a pesar de las condiciones adversas, a la vez que se presentan como una muestra de la capacidad de 
estos profesionales para hacer frente a los problemas de su día a día sin contar, necesariamente, con un apoyo institucional. Así, y aunque algunas de estas experiencias no disponen ni de un tiempo ni de un presupuesto destinados específicamente a ellas, se trata de medidas que, lejos de perseguir indiscriminadamente la quimera de la productividad y la optimización de los recursos, abogan por un cambio en las dinámicas sociales, organizativas y técnicas de los centros de salud, mejorando así la presentación de servicios de estas instituciones.

En suma, consideramos que tanto los gestores públicos como los gerentes de las Áreas de Salud, responsables en última instancia de la organización horaria y presupuestaria de los centros de salud, deberían tener en cuenta los beneficios que la introducción de las TIC puede suponer para mejorar la prestación de servicios sanitarios en estas instituciones. Debemos dar un salto y considerar la asistencia sanitaria no únicamente como un servicio presencial, sino también telefónico y virtual, valorando a los profesionales que lo impulsan y abriendo estas posibilidades al conjunto de la población. Se trata de una apuesta que consiste, en definitiva, en flexibilizar la concepción actual de la consulta médica, extremadamente condicionada por los pocos minutos de que disponen los pacientes para interactuar con los profesionales sanitarios en la consulta presencial, a la vez que aprovechar las posibilidades de que disponen las TIC para mejorar estas condiciones.

\section{Colaboradores}

Martí Oliver-Mora y Lupicinio Iñiguez-Rueda diseñaron el estudio y supervisaron todos los aspectos de su realización. Martí Oliver-Mora realizó el trabajo de campo, el análisis de los resultados y la redacción del artículo. Lupicinio Iñiguez-Rueda revisó críticamente el contenido intelectual del articulo y aprobó su última versión.

\section{Referencias}

1. Gil A, Vall-Ilovera M, Feliu J. Consumo de TIC y subjetividades emergentes: ¿Problemas nuevos? Interv Psicosoc. 2010; 1(19):19-26.

2. Vayreda A, Domènech M. Psicología e internet. Barcelona: Editorial UOC; 2007.

3. Gálvez AM. Sociabilidad en pantalla. Un estudio de la interacción en los entornos virtuales. Rev Antropol Iberoam [Internet]. 2005 [acceso 31 Mar 2016]; 1-20. Disponible en: http://www.redalyc.org/articulo.oa?id=62309916

4. Glaser B, Strauss AL. The discovery of Grounded Theory: strategies for qualitative research. Chicago: Aldine; 2010.

5. Hughes B, Joshi I, Wareham J. Health 2.0 and medicine 2.0: tensions and controversies in the field. J Med Internet Res. 2008; 3(10):e23.

6. O'Reilly T. What is web 2.0: design patterns and business models for the next generation of software [Internet]. 2005 [acceso 31 Mar 2016]. Disponible en: http:// www.oreilly.com/pub/a/web2/archive/what-is-web-20.html

7. Horner K, Wagner E, Tufano J. Electronic consultations between primary and specialty care clinicians: early insights [Internet]. Commonwealth Fund; 2011 [acceso 31 Mar 2016]. Disponible en: http://www.commonwealthfund.org/ /media/files/publications/ issue-brief/2011/oct/1554_horner_econsultations_primary_specialty_care_clinicians_ ib.pdf 
8. Ferguson T. E-patients scholars working group. E-Patients. How they can help us heal health care [Internet]. 2007 [acceso 16 Feb 2015]. Disponible en: http://e-patients.net/ e-Patients_White_Paper.pdf

9. Adahl K. On decision support in participatory medicine supporting health care empowerment. Karlskrona: Blekinge Institute of Technology; 2012.

10. Nutbeam D. Health literacy as a public goal: a challenge for contemporany public health education and communication strategies into the 21 st century. Health Promot Int. 2000; 3(15):259-67.

11. Nutbeam D. The envolving concept of health literacy. Social Sci Med. 2008; (67):2072-8.

12. Eysenbach G. Medicine 2.0: social networking, collaboration, participation, apomediation, and openness. J Med Internet Res [Internet]. 2008 [acceso 31 mar 2016];3(10):e23. Disponible en: http://www.jmir.org/2008/3/e22

13. Emanuel E, Emanuel L. Four models of the physician-patient relationship. J Am Med Assoc. 1992; 16(267):2221-6.

Oliver-Mora M, Iñiguez-Rueda L. O uso das tecnologias da informação e da comunicação (TIC) nos centros de saúde: a visão dos profissionais na Catalunha, Espanha. Interface (Botucatu). 2017; 21(63):945-55.

Esta pesquisa tem por objetivo a identificação de experiências no uso das tecnologias da informação e da comunicação (TIC) que sejam capazes de melhorar o gerenciamento público dos centros de saúde na Catalunha (Espanha), e que sejam impulsionadas "de baixo para cima" por parte dos profissionais da atenção primária. Reunimos estas experiências mediante a realização de onze entrevistas semiestruturadas com profissionais da medicina e enfermagem da atenção primária. O resultado constitui um conjunto de experiências que podem melhorar o gerenciamento público dos centros de saúde por meio de: a) uma maior interação entre pacientes e profissionais da atenção primária; b) uma medicina mais centrada no paciente; e c) uma maior participação e envolvimento dos pacientes em seu estado de saúde.

Palavras-chave: Saúde pública. Centros de saúde. Meios de comunicação. Difusão de inovações. Pesquisa qualitativa. 
